\title{
Reversible Unilateral Abducens Nerve Palsy in a patient with Scrub Typhus - A Clinical Diagnostic Dilemma
}

\section{Rahul Choudhary $^{1}$, Gaurav Katoch ${ }^{1}$, Garima Sachdeva ${ }^{2}$ and Sweta Kushwah ${ }^{3}$}

${ }^{1}$ Department of Paediatrics, NDMC Medical College and Hindu Rao Hospital, Delhi, India

${ }^{2}$ Department of Obstetrics and Gynaecology, NDMC Medical College and Hindu Rao Hospital, Delhi, India

${ }^{3}$ Department of Obstetrics and Gynaecology, Gajra Raja Medical College, Gwalior, Madhya Pradesh, India

\section{Correspondence:}

Garima Sachdeva

Department of Obstetrics and Gynaecology,

NDMC Medical College and Hindu Rao Hospital,

Delhi, India

Email: gsachdeva25@gmail.com

DOI: $10.3126 /$ jnps.v40i3.29468

Submitted on: $2020-06-15$

Accepted on: 2020-10-04

Acknowledgements: None

Funding: Nil

Conflict of Interest: None declared

Permission from IRB: Yes

To cite this article: Choudhary $\mathrm{R}$, Katoch G, Sachdeva G, Kushwah S. Reversible unilateral abducens nerve palsy in a patient with scrub typhus - a clinical diagnostic dilemma J Nepal Paediatr Soc. 2020;40(3): $278-81$.

\begin{abstract}
Scrub typhus is an acute febrile rickettsial disease caused by Orientia tsutsugamushi. It infects endothelial cells and causes vasculitis, the predominant clinico-pathological feature of the disease. This results in disseminated inflammatory perivascular lesions leading to damage to the blood vessels affecting multiple end organs. Abducens nerve palsy is a known but extremely rare and reversible complication of scrub typhus. We present a case of scrub typhus with sixth cranial nerve involvement which responded to treatment with doxycycline.
\end{abstract}

Keywords: Chiggers; Doxycycline; Rickettsia; Orientia Tsutsugamushi; Scrub Typhus; Trombiculid mites

This work is licensed under creative common attribution 3.0 license 


\section{INTRODUCTION}

Scrub typhus is an acute febrile rickettsial disease caused by gram-negative obligate intracellular parasite "Orientia tsutsugamushi." It proliferates within the endothelial cells of the blood vessels and causes vasculitis, the predominant clinicopathological feature of the disease. ${ }^{1}$ Scrub typhus presents with variable clinical manifestations which may range from simple flulike illness to potentially life-threatening multiorgan failure.

Central nervous system (CNS) involvement in scrub typhus manifests as the presence of meningitis, encephalomyelitis, eighth cranial nerve involvement resulting in deafness and peripheral neuropathy. ${ }^{2}$ Abducens nerve palsy is very rarely associated with scrub typhus. Here we present a case of unilateral reversible abducens nerve palsy in a child with scrub typhus which resolved completely following treatment. Also, an attempt has been made to review the clinical presentation, complications, diagnosis, treatment, and preventive measures of the disease.

\section{CASE REPORT}

A 13 year old developmentally normal girl from a village in North India presented to our institute with complaint of fever. The fever was there for seven days, followed by vomiting for four days, headache for four days and double vision and medial deviation of the right eye for two days. There was also a history of multiple painful neck swellings four to five days before the onset of fever. The patient did not give any history of head injury or any recent vaccinations. The girl had normal birth and past history.

On examination, the child was conscious, oriented, and cooperative with stable vitals. There was no eschar. On ophthalmological examination, right esotropia was present. Lateral gaze was not possible in the right eye (Figures 1-3). There were no meningeal signs, the fundus was normal. Rest of the CNS examination was normal. Other organ systems examination including respiratory, cardiovascular, and gastrointestinal system was within normal limits.
Laboratory investigations revealed moderate anaemia with haemoglobin of $10.2 \mathrm{gm} / \mathrm{dl}$, mildly elevated leukocyte count of $12,000 / \mathrm{mm}^{3}$, with a differential count of $72 \%$ neutrophils and $24 \%$ lymphocytes. Platelet count was on the lower side $\left(98,000 / \mathrm{mm}^{3}\right)$. ESR was $43 \mathrm{~mm}$ in first hour. Peripheral blood film showed normocytic normochromic anaemia. Liver function tests showed elevated bilirubin, raised aspartate transaminase (AST) $(210 \mathrm{IU} / \mathrm{L})$, alanine transaminase (ALT) (150 IU/L), and alanine phosphatase (ALP) (170 IU/L). Lumbar puncture was done due to a history of high-grade fever and headache. Cerebrospinal fluid (CSF) examination revealed lymphocytic pleocytosis [White blood count (WBC) of 12 cells $/ \mathrm{mm}^{3}, 100 \%$ lymphocytes], CSF glucose level was $48 \mathrm{mg} / \mathrm{dl}$ and protein $76 \mathrm{mg} /$ dl. CSF gram stain, ZN stain, and culture reports were negative. Montoux test was non-reactive and chest $\mathrm{x}$-Ray and MRI brain were normal. Dengue NS-1 antigen and IgG, IgM anti-dengue antibodies by ELISA were negative. Peripheral blood film was negative for malarial parasite. Widal's test was negative. Since the child was from endemic region, IgM for scrub typhus was done and it came out positive.

During the hospital stay, the patient was managed symptomatically for fever and vomiting. Doxycycline was given for six days and was discharged on the $7^{\text {th }}$ day of admission and was advised to continue doxycycline for four more days. Fever and vomiting subsided and the child improved symptomatically by third day of admission. However, her lateral rectus palsy persisted which resolved when she came for follow up after one week.

\section{DISCUSSION}

Scrub typhus is particularly endemic in areas where secondary growth of scrub vegetation occurs after clearing of the forests. This patient was residing in North India, which is known to be endemic for scrub typhus infection.

Initial clinical manifestations of scrub typhus are usually vague like in our patient. The common initial non-specific manifestations include fever, rash, headache, myalgia, nausea, vomiting, 


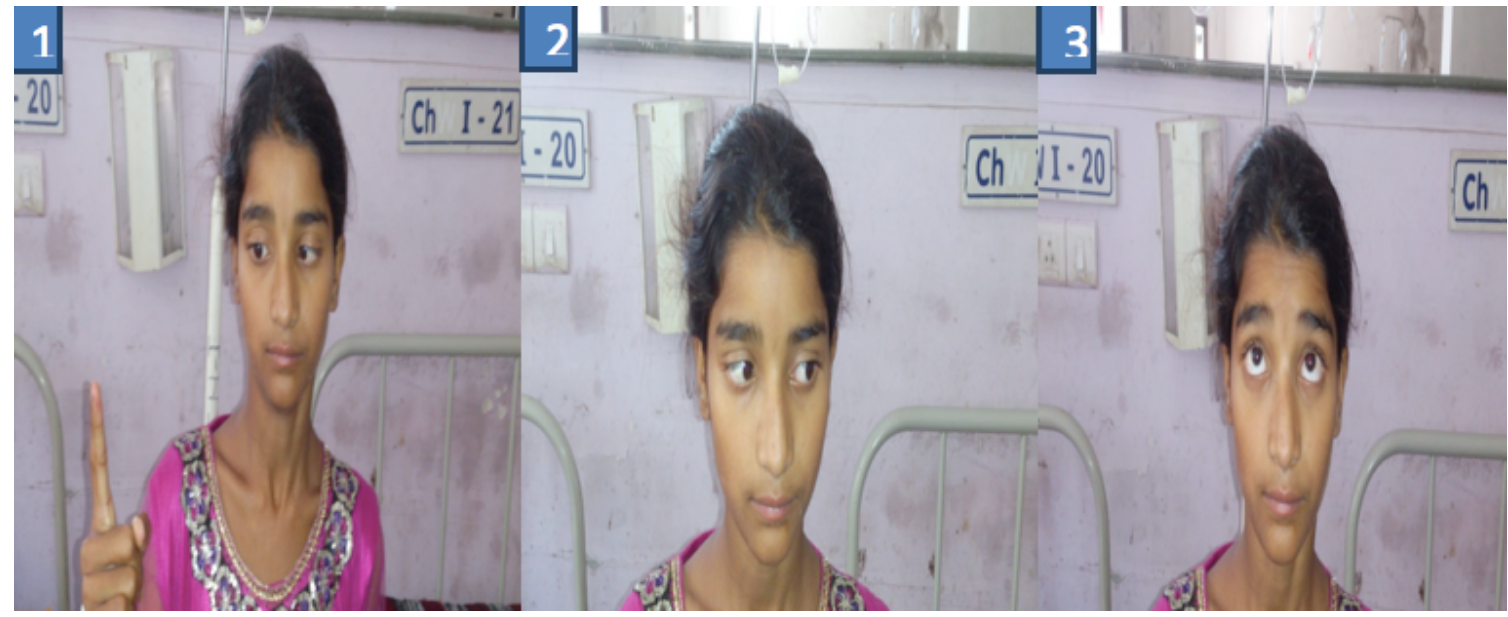

Figure 1. Limited abduction in the right eye

Figure 2. Normal adduction of the right eye

Figure 3. Normal elevation of right eye

abdominal pain, cough, and generalised lymphadenopathy. ${ }^{1}$

It is transmitted by the bite of larval trombiculid mites (chiggers) inhabiting the scrub vegetation. ${ }^{3}$ Multiplication of the organism at the site of the bite results in the characteristic eschar. It initially begins as a papule at the site of the bite and then ulcerates forming a black crust-like cigarette lesion, a central black crust with erythema around its periphery. ${ }^{4}$ It is mostly non-pruritic and painless. Hence, it is most often missed by the patients. The average reported prevalence of eschar is around 7 to $87 \%$ in patients with scrub typhus. ${ }^{4}$ No eschar was noted in our patient. However, if present, eschar is a very valuable clue towards scrub typhus and helps in early diagnosis and initiation of treatment. So, one should diligently look for its presence.

Although, scrub typhus is known to involve the central nervous system, isolated unilateral abducens nerve palsy is a very rare complication. The proposed pathogenesis for the same is due to microinfarction of vasa vasorum of the nerve, resulting in the stretching of the vessels. ${ }^{5}$ In the present case, sudden onset abducens nerve palsy was noted and its occurrence coincided with the peak of the symptoms. Moreover, complete resolution occurred once the treatment was initiated. Other cranial nerves reported in the literature to be associated with scrub typhus are eighth cranial nerve resulting in sensorineural hearing loss and facial nerve resulting in facial nerve palsy. 6,7

Doxycycline is the drug of choice for treating scrub typhus. It is orally effective in mild cases, but severe cases generally require parenteral treatment. Other antibiotics that are effective include tetracycline, azithromycin and telithromycin. Rifampicin is prescribed in resistant cases. ${ }^{8}$ Our patient responded to doxycycline with complete resolution of symptoms and abducens nerve palsy within two weeks.

In this patient, although the classical eschar was not present, several pointers were indicating the diagnosis of scrub typhus. The patient belonged to the endemic area. There was a sudden onset abducens nerve palsy which completely resolved on doxycycline intake. Hence, one should keep in mind the differential diagnosis of scrub typhus while treating such patients. Abducens nerve palsy in this patient developed when the patient was maximally symptomatic. Moreover, complete resolution was seen once the patient was treated with doxycycline. Hence, it can be concluded that this event was most likely secondary to scrub typhus infection.

\section{CONCLUSIONS}

When a patient from the endemic area presents with the flu-like symptoms and unusual presentation like in this case abducens nerve palsy, 
with or without the presence of eschar, it should alert the physicians to suspect the possibility of scrub typhus. It is also important to note that central nervous system involvement with scrub typhus including cranial nerve deficits are easily reversible without any permanent sequelae with timely diagnosis and with appropriate antibiotics such as doxycycline, chloramphenicol, and azithromycin.

\section{REFERENCES}

1. Rapsang AG, Bhattacharyya P. Scrub typhus. Indian J Anaesth. 2013;57(2):127-34.DOI: 10.4103/0019-5049.111835

2. Pai H, Sohn S, Seong Y, Kee S, Chang WH, Choe KW. Central nervous system involvement in patients with scrub typhus. Clin Infect Dis. 1997;24(3):436-40. DOI: 10.1093/clinids/24.3.436

3. Elliott I, Pearson I, Dahal P, Thomas NV, Roberts T, Newton PN. Scrub typhus ecology: a systematic review of Orientia in vectors and hosts. Parasit Vectors. 2019;12(1):513. DOI: 10.1186/s13071-019-3751-X

4. Rose W, Rajan RJ, Punnen A, Ghosh U. Distribution of Eschar in Pediatric Scrub Typhus. J Trop Pediatr. 2016;62(5):415-20. DOI: 10.1093/tropej/fmw027

5. Mahajan SK. Neuropsychiatric Manifestations of Scrub Typhus. J Neurosci Rural Pract. 2017;8(3):421-6. DOI: 10.4103/jnrp.jnrp_44_17

6. Kang JI, Kim DM, Lee J. Acute sensorineural hearing loss and severe otalgia due to scrub typhus. BMC Infect Dis. 2009;9:173. DOI: $10.1186 / 1471-2334-9-173$

7. Lin WR, Chen TC, Lin CY, Lu PL, Chen YH. Bilateral simultaneous facial palsy following scrub typhus meningitis: a case report and literature review. Kaohsiung J Med Sci. 2011;27(12):573-6. DOI: 10.1016/j.kjms. 2011.10.003

8. Rajapakse S, Rodrigo C, Fernando SD. Drug treatment of scrub typhus. Trop Doct. 2011;41(1):1-4. DOI: 10.1258/ td.2010.100311 